\title{
The impact of the absence of glycopeptidolipids on the ultrastructure, cell surface and cell wall properties, and phagocytosis of Mycobacterium smegmatis
}

\author{
Gilles Etienne, ${ }^{1}$ Christelle Villeneuve, ${ }^{1}$ Helen Billman-Jacobe, ${ }^{2}$ \\ Catherine Astarie-Dequeker, ${ }^{1}$ Marie-Ange Dupont ${ }^{3}$ \\ and Mamadou Daffé ${ }^{1}$
}

1 Institut de Pharmacologie et de Biologie Structurale, Unité Mixte de Recherche du Centre de National de Recherche Scientifique et de l'Université Paul Sabatier (UMR 5089), 205 route de Narbonne, 31077 Toulouse cedex 04 France

2 Department of Microbiology and Immunology, University of Melbourne, Victoria 3010 Australia

3 Institut d'Exploration Fonctionnelle des Génomes (IFR 109), 118 route de Narbonne, 31062 Toulouse cedex France
Author for correspondence: Mamadou Daffé. Tel: +33 561175 569. Fax: +33 561175580 . e-mail:mamadou.daffe@ipbs.fr

Glycopeptidolipids (GPLs) are a class of species- or type-specific mycobacterial lipids and major constituents of the cell envelopes of many non-tuberculous mycobacteria. To determine the function of GPLs in the physiology of these bacteria, a mutant of Mycobacterium smegmatis in which the gene encoding a mycobacterial nonribosomal peptide synthetase has been inactivated by transposon mutagenesis was analysed. Labelling experiments indicated that half of the bacterial GPLs were located on the cell surface and represented $85 \%$ of the surface-exposed lipids of the parent strain whereas the mutant was defective in the production of the GPLs. Compared to the parent smooth morphotype strain, the GPL-deficient mutant strain exhibited a rough colony morphology, an increase of the cell hydrophobicity and formed huge aggregates. As a consequence, the mutant cells were no longer able to bind ruthenium red, as observed by transmission electron microscopy. The altered surface properties of the mutant cells also affected the phagocytosis of individual bacilli by human monocyte-derived macrophages since mutant cells were internalized more rapidly than cells from the parent strain. Nevertheless, no specific release of surface constituents into the culture broth of the mutant was observed, indicating that the cell surface is composed of substances other than GPLs and that these are essential for maintaining the architecture of the outermost layer of the cell envelope. Importantly, the absence of these major extractable lipids of $M$. smegmatis from the mutant strain has a profound effect on the uptake of the hydrophobic chenodeoxycholate by cells, indicating that GPLs are involved in the cell wall permeability barrier of $M$. smegmatis. Altogether, these data showed that, in addition to being distinctive markers of numerous mycobacterial species, GPLs play a role in the bacterial phenotype, surface properties and cell wall permeability.

Keywords: mycobacteria, cell wall permeability, morphology, ultrastructure, glycolipids

\section{INTRODUCTION}

The mycobacterial cell envelope, as revealed by the most recent developments in knowledge of the ultrastructure and chemistry of mycobacteria, is composed of three

Abbreviations: GPL, glycopeptolipid; MDM, monocyte-derived macrophage; OL, outer layer; SEM, surface-exposed material. structural components: a plasma membrane, a wall and an outer layer (OL) (Daffé \& Draper, 1998). While the plasma membrane appears to be a typical bacterial membrane, the complex wall that surrounds this membrane partly resembles a Gram-positive wall, but is unusual in having the thick layer of peptidoglycan covalently linked to a D-arabino-D-galactan, which in turn is esterified by long-chain fatty acids, the so-called 


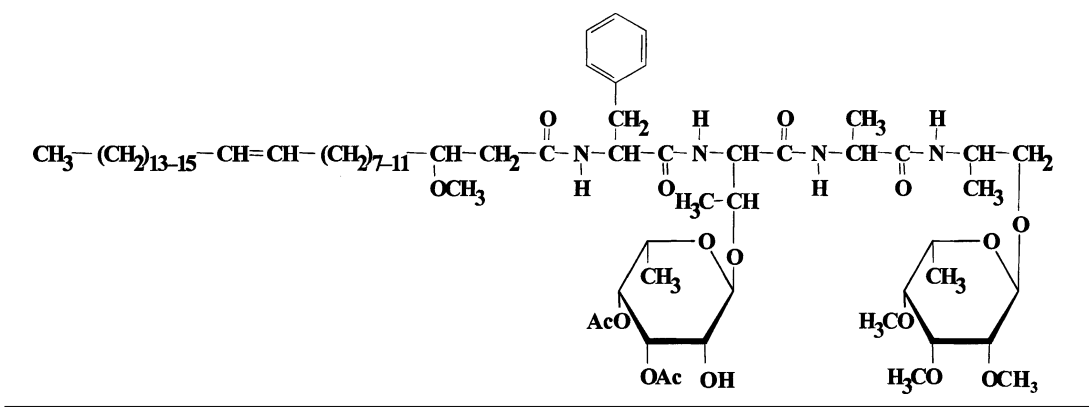

Fig. 1. Structure of the major glycopeptidolipids of M. smegmatis. Ac, acetyl. mycolic acids. Unlike Gram-positive walls, the mycobacterial wall also contains a great variety of other lipids, which are probably arranged to form an asymmetric bilayer with the cell wall mycolate monolayer, but not covalently attached to it (Minnikin, 1982). In addition, pore-forming proteins, typifying Gram-negative bacteria, have been characterized in the walls of several mycobacterial species (Mukhopadhyay et al., 1997; Trias et al., 1992, Trias \& Benz, 1994; Senaratne et al., 1998). Consequently, although mycobacteria are phylogenetically classified as Gram-positive bacteria, their wall is more related to that of Gram-negative micro-organisms (Draper, 1998). The outer layer of the cell envelope of mycobacteria consists of a proteincarbohydrate matrix loosely bound to the wall and contains both ubiquitous and species-specific lipids (Daffé \& Draper, 1998). Many distinctive features of mycobacteria have been associated with the uncommon composition of their cell envelope. For instance, the well-known acid-fastness of mycobacteria and relatives, i.e. the failure of dilute acids to decolourize bacteria stained with various basic dyes, may be explained by the presence of the asymmetric bilayer, which would represent a permeability barrier to polar molecules (Brennan \& Nikaido, 1995). The existence of this barrier could also explain their limited permeability and intrinsic resistance to strong chemicals such as acids, alkalis and hypochlorite (Daffé \& Draper, 1998). Likewise, the unusual composition of the mycobacterial cell surface, particularly its remarkable hydrophobicity, may explain the tendency of cells to grow in large aggregates.

The high lipid content of mycobacterial cells, recognized as early as the 1930s, and the extraordinary biological activities associated with some of the purified lipids (for reviews see Daffé \& Draper, 1998; Goren \& Brennan, 1979), have prompted researchers to devote much effort to the identification of the various types of mycobacterial lipids. Among the characteristic lipids of mycobacteria are the species-specific glycopeptidolipids (GPLs) that typify many non-tuberculous mycobacterial species; these molecules may be subdivided into alkali-stable Ctype GPLs and alkali-labile serine-containing GPLs (Daffé \& Lemassu, 2000). The C-type GPLs have been found in saprophytic mycobacteria (Mycobacterium smegmatis), opportunistic pathogens of man (Mycobacterium avium-intracellulare, Mycobacterium scrofulaceum, Mycobacterium peregrinum, Mycobacterium chelonae, Mycobacterium abscessus) and of animals (Mycobacterium lepraemurium, Mycobacterium paratuberculosis, Mycobacterium porcinum, Mycobacterium senegalense) (Brennan, 1988; Daffé \& Lemassu, 2000) whereas the alkali-labile serine-containing GPLs have been found so far only in Mycobacterium xenopi (Besra et al., 1993; Rivière \& Puzo 1991). C-type GPLs share a common lipopeptidyl core consisting of a mixture of 3-hydroxy and 3-methoxy $\mathrm{C}_{26-34}$ fatty acids (Daffé et al., 1983) amidated by a tripeptide D-Phe-Dallo-Thr-D-Ala and terminated by L-alaninol (Fig. 1). They differ from one another by the number and the nature of the saccharide units linked to the hydroxyl group of allo-Thr and/or alaninol (Brennan, 1988; Daffé \& Lemassu, 2000). These glycosyl units are responsible for both the variability and the specificity of the antigenically distinct serovariants within the M. aviumintracellulare complex and several other C-type GPLcontaining mycobacteria (Brennan, 1988), which implies that these compounds occur on the peripheral bacterial surface, in agreement with their isolation from the outermost compartment of mycobacterial cells (OrtaloMagné et al., 1996) and their identification as the receptor of mycobacteriophage D4 (Furuchi \& Tokunaga, 1972; Goren et al., 1972). Although the role of GPLs in pathogenesis is still unclear (Daffé \& Draper, 1998), they accumulate into the phagosome during bacterial intracellular growth, contributing to the formation of a capsule around the bacteria (Draper, 1974; Rulong et al., 1991; Tereletsky \& Barrow, 1983). Less is known, however, about the localization of GPLs in deeper compartments of the mycobacterial cell envelope and their possible contribution to the cell wall permeability barrier. Thanks to the availability of $M$. smegmatis mutants with transposon insertions in the genes involved in the synthesis of the C-type GPL core (Billman-Jacobe et al., 1999; Recht et al., 2000), we addressed the question of the effects of the absence of C-type GPLs on the architecture and surface properties of the cell envelope of M. smegmatis and on the initial interactions of bacteria with host cells.

\section{METHODS}

Bacterial strains and culture. M. smegmatis $\mathrm{mc}^{2} 155$ and its isogenic GPL-deficient mutant strains TM99, 8-245 and 9-1045 (Billman-Jacobe et al., 1999; Recht et al., 2000) were routinely grown at $37^{\circ} \mathrm{C}$ with shaking at 250 r.p.m. (Incubator Shaker, New Brunswick Scientific) in Luria-Bertani (LB broth, Euromedex) medium with the addition of $0.05 \%$ Tween 80 . 
The strains were also grown on Trypticase-Soy (TS broth, bioMérieux) medium for the determination of surface properties of the cells and on Sauton's medium, without agitation, as surface pellicles when information was needed on extracellular materials. The mutant strains were maintained by kanamycin selection, with kanamycin added to a final concentration of $25 \mu \mathrm{g} \mathrm{ml}^{-1}$. The generation times of the various strains were determined as described by Raynaud et al. (1998) by weighing cells grown in the LB medium.

The viability of mycobacterial cells was assessed by i) quantifying the level of isocitrate dehydrogenase, an indicator of autolysis, in the culture filtrates (Raynaud et al., 1998) and ii) labelling cells with both propidium iodide and fluorescein diacetate (Cougoule et al., 2002); the percentages of live (fluorescein-positive, green cells) and dead (propidium iodide, red cells) bacteria were determined by counting at least 100 bacteria.

Single-cell suspensions were prepared with late exponentialphase cultures incubated in $100 \mathrm{ml}$ Sauton's medium; the pellicles were harvested by pouring off the medium and were gently shaken for $30 \mathrm{~s}$ with 5 g glass beads $(4 \mathrm{~mm}$ diameter). The declumped cells resulting from this treatment were suspended in $10 \mathrm{ml}$ TS broth plus $0.05 \%$ Tween 80 and centrifuged for $10 \min 100 \mathrm{~g}$. The $\mathrm{OD}_{650}$ of the supernatant, which contains mainly single cells, was adjusted to $1 \mathrm{OD}_{650}$ unit and directly used to inoculate the media (100 $\mu$ l suspension for $50 \mathrm{ml}$ broth, except where indicated otherwise) for the determination of some cell surface properties. Alternatively cells were washed three times with PBS to give a PBS-washed single cell suspension, or stored at $-80^{\circ} \mathrm{C}$ in the presence of $20 \%$ (w/v) glycerol.

MICs were determined by the agar two-fold dilution method in TS agar (bioMérieux). The drug-containing media were inoculated with $100 \mu \mathrm{l}$ of a dilution $\left(0 \cdot 01 \mathrm{OD}_{650}\right.$ unit) of the single cell suspension stored at $-80^{\circ} \mathrm{C}$ and incubated for 7 days at $37^{\circ} \mathrm{C}$. For all the drugs tested, $99 \%$ inhibition of bacterial growth was determined as the MIC of the drug.

Motility. Surface-spreading assays were adapted from Martinez et al. (1999); briefly, Middlebrook 7H9 base medium (Difco) was solidified with $0.4 \%$ high grade agarose (Eurogentec). Plates were inoculated into their centre with $10 \mu$ of the $1 \mathrm{OD}_{650}$ unit PBS-washed single cell suspension (see above). Spreading was evaluated after incubation for 5 days at $37^{\circ} \mathrm{C}$ in a humidified incubator by measuring the diameter of the halo of growth formed by the mycobacteria.

Cellular aggregation. Mycobacterial strains were cultivated with shaking ( 250 r.p.m.) in TS broth without Tween 80 for 3 days at $37^{\circ} \mathrm{C}$. The bacterial suspensions were centrifuged for $10 \mathrm{~min}$ at $100 \mathrm{~g}$ to separate the unicellular mycobacteria from the aggregates, which were pelleted (Cougoule et al., 2002). The single cells were further recovered by centrifugation for $30 \mathrm{~min}$ at $3000 \mathrm{~g}$. Cell pellets were dried and weighed, and the cellular aggregation was calculated, i.e. the percentage of aggregate-containing pellets versus total cell weight.

Congo red accumulation. The assay (Cangelosi et al., 1999) was adapted as follows: mycobacteria were cultivated for 3 days at $37^{\circ} \mathrm{C}$ with shaking (250 r.p.m.) in TS broth plus $100 \mu \mathrm{g}$ Congo red $\mathrm{ml}^{-1}$ and $0 \cdot 05 \%$ Tween 80 . Cells were collected by centrifugation ( $30 \mathrm{~min}$ at $3000 \mathrm{~g}$ ) and washed extensively with distilled water until the supernatant was colourless. Cells were resuspended in $1 \mathrm{ml}$ acetone, vortexed and gently shaken for $2 \mathrm{~h}$ at room temperature; cells were then removed by highspeed microcentrifugation and Congo red in the supernatants was spectrophotometrically measured at $488 \mathrm{~nm}$. The Congo red binding was defined as the $A_{488}$ of the acetone extracts divided by the dry weight (in $\mathrm{mg}$ ) of the cell pellet.

Hydrophobicity index. Relative hydrophobicities were assessed by the hexadecane partition procedure (Rosenberg et al., 1980). Briefly, $1 \mathrm{OD}_{650}$ unit PBS-washed single cell suspension (see above) of each strain was mixed with $0.3 \mathrm{ml}$ hexadecane (Avocado) by vortexing for $2 \mathrm{~min}$. The hydrophobicity index was defined as the percentage reduction in the $\mathrm{OD}_{650}$ of the aqueous phase; this reduction was determined for triplicate samples after allowing $15 \mathrm{~min}$ for the hydrocarbon phase to rise completely.

Zeta potential. For determination of the bacterial cell surface charge (Bayer \& Sloyer, 1990), zeta-potential ( $\zeta$ ) measurements were performed with $1 \mathrm{OD}_{650}$ unit PBS-washed single cell suspension (see above) in a zetameter Zetasizer 3000 (Malvern Instruments).

Isolation, fractionation and analysis of the extracellular and surface-exposed components. Surface-exposed material (SEM) and extracellular compounds were extracted and analysed as previously described (Lemassu et al., 1996; OrtaloMagné et al. 1996). Briefly, mycobacterial cells were harvested by centrifugation $(30 \mathrm{~min}$ at $3000 \mathrm{~g}$ ) and the culture broths were sterilized by filtration through $0 \cdot 20 \mu \mathrm{m}$ pore-size sterile filters (Nalgene). Cells were shaken for $1 \mathrm{~min}$ with $10 \mathrm{~g}$ glass beads (4 mm diameter) per $2 \mathrm{~g}$ (wet weight) cells (OrtaloMagné et al., 1995), resuspended in distilled water $(50 \mathrm{ml}$ per flask) and then the cells were removed by filtration. A portion of the crude filtrate, which contains the SEMs, and of the culture broths, which contains the extracellular materials, were concentrated separately under vacuum, extensively dialysed against distilled water and analysed by colorimetric assays for their carbohydrate content (Dische, 1962) and protein content by the Lowry method. Chloroform and methanol were added to the remaining portions to obtain partition mixtures composed of chloroform/methanol/water (3:4:3, by vol.); the organic phases were concentrated, washed with water, evaporated to dryness to yield crude lipid extracts, and weighed. The lipid extracts were dissolved in chloroform and analysed by TLC on silica gel Durasil 25precoated plates $(0 \cdot 25 \mathrm{~mm}$ thickness; Macherey-Nagel). The lipids were resolved by TLC run in the following solvent mixtures: petroleum ether/diethyl ether $(9: 1, \mathrm{v} / \mathrm{v})$ for analysing triacyl glycerols, chloroform/methanol $(9: 1, \mathrm{v} / \mathrm{v})$ for GPLs and trehalose dimycolates and chloroform/methanol/ water (60:30:8 by vol.) for trehalose monomycolates and phospholipids. Sugar-containing compounds (GPLs, trehalose dimycolates, trehalose monomycolates and phosphatidylinositol mannosides) were visualized by spraying the plates with $0 \cdot 2 \%$ anthrone in concentrated sulfuric acid, followed by heating at $110{ }^{\circ} \mathrm{C}$. The Dittmer-Lester reagent (Dittmer \& Lester, 1964) was used to detect phosphorus-containing substances. The ninhydrin reagent was used to reveal the presence of free amino groups and a spray with $10 \%$ molybdophosphoric acid in ethanol solution, followed by heating at $110^{\circ} \mathrm{C}$, was used to detect all of the lipid spots, including triacyl glycerols.

Labelling of lipids. The various classes of extractable and cell surface lipids were quantified by labelling; $1.2 \mathrm{MBq}$ sodium $\left[{ }^{14} \mathrm{C}\right]$ acetate (Amersham) was added to $100 \mathrm{ml}$ 2-day-old cultures containing mid-exponential-phase bacteria. After $16 \mathrm{~h}$ incorporation, the reaction was stopped by centrifugation and the SEMs were isolated by extraction with glass beads (Ortalo-Magné et al., 1995). Lipids from these latter materials and those from bead-treated cells were extracted with chloroform/methanol $(1: 2, \mathrm{v} / \mathrm{v})$. Both types of lipid extracts 
were analysed by TLC using the solvent mixtures described above; the radioactivity was located and counted on plates using an automatic TLC linear analyser (Berthold LB 2832). Then the lipid spots were visualized by spraying with the appropriate reagents, with heating when necessary.

Permeability assays. The permeability of the strains of $M$. smegmatis to chenodeoxycholate was assessed as previously described (Bardou et al., 1998; Jackson et al., 1999). Exponentially grown cells (2-day-old cultures) were first labelled for $16 \mathrm{~h}$ with $\left[5,6{ }^{3} \mathrm{H}\right]$ uracil $\left(20 \mu \mathrm{M}, 1.85 \mathrm{GBq} \mathrm{mmol}^{-1}\right.$; DuPont NEN) to quantify the biomass present in aliquots used in the accumulation assays. Then, cells were collected by centrifugation and washed with $10 \mathrm{mM}$ HEPES, pH 7.2. Aliquots of labelled cells were counted, dried and weighed to correlate ${ }^{3} \mathrm{H}$ labelling with cell dry weight. Accumulation assays were performed under continuous agitation. $\left[{ }^{14} \mathrm{C}\right]$ chenodeoxycholate $\left(20 \mu \mathrm{M}, 1.8 \mathrm{GBq} \mathrm{mmol}^{-1}\right.$; DuPont $\mathrm{NEN}$ ) was added to a $1 \mathrm{ml}$ mixture of HEPES containing about $40 \mathrm{mg}{ }^{3} \mathrm{H}$-labelled cells. Aliquots $(0 \cdot 1 \mathrm{ml})$ were removed at different time intervals and added to the top of an Eppendorf centrifuge tube containing $0.25 \mathrm{ml}$ silicon oil/paraffin oil $(1: 0 \cdot 2, \mathrm{v} / \mathrm{v})$. Cells were separated from the accumulation medium by centrifugation $(13000 \mathrm{~g}, 1 \mathrm{~min})$. Centrifuged tubes were frozen on dry ice and the pellets were dropped into counting flasks by cutting the cone top. Then the scintillation solution (Aqualuma) was added and the vials were sonicated for $30 \mathrm{~min}$ in a water bath to disperse cells.

Transmission electron microscopy. The method for preparation of samples for transmission electron microscopy was based on the procedures of Paul \& Beveridge (1992). M. smegmatis $\mathrm{mc}^{2} 155$ and the GPL-deficient mutant TM99 were grown for $72 \mathrm{~h}$ in both the LB broth and Sauton's medium, then subcultured into fresh broths by making a 1/100 dilution. The fresh broth was incubated overnight and cells were harvested by centrifugation $(8000 \mathrm{~g}, 10 \mathrm{~min})$, and washed twice in PBS. The resulting pellets were fixed in $2.5 \%(\mathrm{w} / \mathrm{v})$ glutaraldehyde, $0.05 \%(\mathrm{w} / \mathrm{v})$ ruthenium red in cacodylate buffer for $2 \mathrm{~h}$ in the dark at room temperature. Cells were washed three times in cacodylate buffer $(0.1 \mathrm{M}, \mathrm{pH} 6 \cdot 8)$, postfixed for $2 \mathrm{~h}$ in the dark in $1 \%(\mathrm{w} / \mathrm{v})$ osmium tetroxide, $0.05 \%(\mathrm{w} / \mathrm{v})$ ruthenium red and then washed twice each in cacodylate buffer and in water. Cells were dehydrated through a graded ethanol series of $10,20,30,40,50,60,70,80$ and $95 \%$ for $5 \mathrm{~min}$ each then washed twice for $15 \mathrm{~min}$ each in $100 \%$ ethanol, then twice for $15 \mathrm{~min}$ each in propylene oxide. Cells were suspended in $1: 1$ propylene oxide/Spurr resin for $2 \mathrm{~h}$ in an open tube. After infiltration overnight, the tubes were opened for $2 \mathrm{~h}$; then samples were transferred to $100 \%$ Spurr resin and left overnight. Resin was replenished the next morning and samples were left to cure at $60^{\circ} \mathrm{C}$ overnight. Blocks were thin-sectioned on a Reichert-Jung microtome and mounted on copper grids. Sections were poststained with uranyl acetate and Reynold's lead citrate. Microscopy was performed on a JEOL 120 EX electron microscope.

Phagocytosis. Macrophages were obtained by isolating monocytes from human peripheral blood as previously described by Astarie-Dequeker et al. (1999); these monocyte-derived macrophages (MDMs) were cultured at $37^{\circ} \mathrm{C}$ in $5 \% \mathrm{CO}_{2}$ for 6 to 7 days on sterile glass coverslips in 24-well tissue culture plates $\left(5 \times 10^{5}\right.$ cells per well) containing RPMI medium supplemented with $10 \%$ inactivated foetal calf serum and antibiotic; the culture medium was renewed on the third day. Before use, MDMs were washed twice with fresh serum-free RPMI and equilibrated for $20 \mathrm{~min}$ at $37^{\circ} \mathrm{C}$ in $5 \% \mathrm{CO}_{\text {. }}$. Single cells of $M$. smegmatis were prepared and fluorescently labelled with FITC as previously described (N'Diaye et al., 1998). MDMs were incubated with $M$. smegmatis in serum-free RPMI, for the time indicated, and then washed twice with fresh medium to remove unbound particles. Phagocytosis of FITC-stained bacteria was determined as previously described (Peyron et al., 2000). Briefly, MDMs were fixed with 3.7\% paraformaldehyde in PBS containing $15 \mathrm{mM}$ sucrose, $\mathrm{pH} 7 \cdot 4$, for $20 \mathrm{~min}$ at room temperature. After neutralization with $50 \mathrm{mM} \mathrm{NH}_{4} \mathrm{Cl}$, extracellular mycobacteria were labelled with rabbit polyclonal antibodies directed against mycobacteria $(1 / 50)$, revealed by TRITC-conjugated secondary antibodies. MDMs containing at least one FITC-stained bacterium $(\sim 100$ cells) were counted in duplicate samples.

\section{RESULTS}

\section{Phenotypic characterization of a GPL-deficient mutant}

The TM99 mutant strain is derived from M. smegmatis $\mathrm{mc}^{2} 155$ by transposon insertion mutagenesis (BillmanJacobe et al., 1999). Genetic analysis revealed that this phenotype was the result of the insertion of a transposon in the mps gene encoding a mycobacterial nonribosomal peptide synthetase, while lipid compositional analysis indicated that the strain was defective in the production of GPLs (Billman-Jacobe et al., 1999). Strain TM99 displayed a rough colony morphology, compared to the smooth aspect of the $\mathrm{mc}^{2} 155$ parent strain (Fig. 2); these data were consistent with previous observations that showed a correlation between the absence of C-type GPL and the rough morphotype (Barrow \& Brennan, 1982; Belisle et al., 1991; Recht et al., 2000).

When the motility of the strains on agar plates were compared (Table 1), the TM99 strain was found to display a spreading halo lower than that of the parent $\mathrm{mc}^{2} 155$ strain. Knowing that the diameter of the spreading halo is highly susceptible to the experimental conditions, depending particularly on the moisture at the surface of the medium, the above result was better expressed in terms of relative motility; accordingly, the relative motility of the mutant strain was consistently three- to fivefold smaller than that of the parent strain. The mutant strain exhibited also identical macroscopic aspects in broth cultures, both in shaken and static (in pellicles) growth conditions : under agitation and even in the presence of Tween 80, the mutant grew as huge aggregates, which became perceptible to the naked eye within 3 days, whereas $\mathrm{mc}^{2} 155$ rarely displayed aggregates, visible only by microscopy and composed of 3 to 5 cells. In addition, while the parent strain formed thin (1 mm height) pellicles that could hardly persist for more than 4 days on Sauton's medium, cells from the mutant strain were heavily clumped and gave thick and robust (3-5 $\mathrm{mm}$ height) pellicles that persisted at the surface of the medium for more than 10 days. These phenotypic changes were neither attributable to the growth rates of the strains, since they exhibited a similar generation time $(3 \cdot 5-4 \mathrm{~h}$ in LB medium), nor to cell lysis. These data indicated that the absence of GPL resulted in marked effects on the surface properties of the mutant cells. 

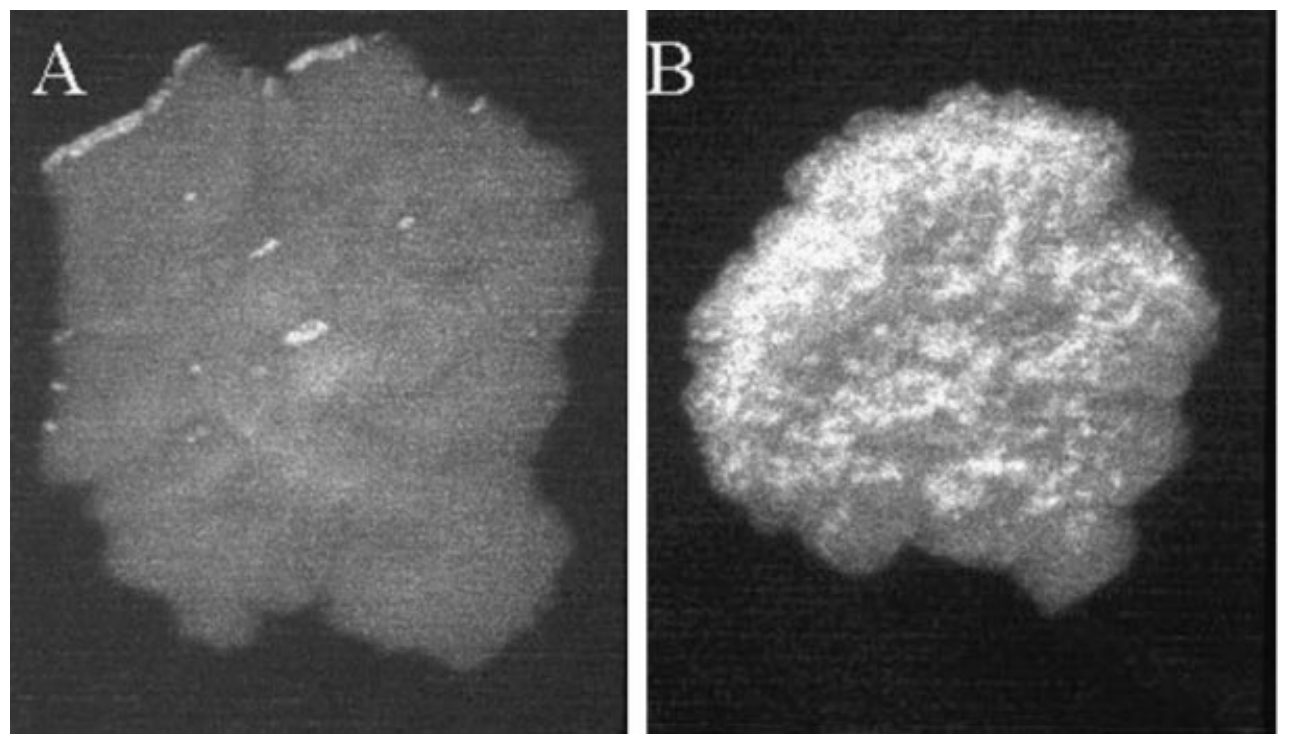

Fig. 2. Colony morphology of $M$. smegmatis $\mathrm{mc}^{2} 155(\mathrm{~A})$ and its isogenic mps-inactivated mutant TM99 (B) on Luria agar.

Table 1. Phenotypic and surface properties of the wildtype $\left(\mathrm{mc}^{2} 155\right)$ and $m p s$-inactivated (TM99) strains of $M$. smegmatis

Results are expressed as mean \pm SD of at least three independent experiments.

\begin{tabular}{|lcc|}
\hline Surface properties & mc $^{2} 155$ & TM99 \\
\hline Motility* & $29 \cdot 1 \pm 18 \cdot 7$ & $8 \cdot 7 \pm 1 \cdot 9 \#$ \\
Cellular aggregation $\dagger$ & $20 \cdot 1 \pm 3 \cdot 1$ & $97 \cdot 9 \pm 0 \cdot 4 \#$ \\
Congo red binding $\neq$ & $211 \pm 81$ & $439 \pm 979$ \\
Hydrophobicity index $\$ & $34 \cdot 9 \pm 19 \cdot 7$ & $51 \cdot 7 \pm 18 \cdot 5 \#$ \\
Zeta potential $\|$ & $-27 \cdot 6 \pm 1 \cdot 2$ & $-28 \cdot 1 \pm 3 \cdot 6$ \\
\hline
\end{tabular}

*Diameter (in $\mathrm{mm}$ ) of the halo formed.

† Percentage of aggregated cells.

$\neq 10^{-4} \times A_{488}$.

$\$$ Arbitrary units, expressed as the percentage of cells recovered in the organic phase.

$\|$ Expressed in $\mathrm{mV}$.

I $P<0.05$ when compared with control calculated with paired $\# P<0.01\} \begin{aligned} & \text { (motility and hydrophobicity) or unpaired Student's } \\ & t \text { test. }\end{aligned}$

\section{Cell surface properties of the mps-inactivated strain TM99}

To tentatively quantify the aggregation level of the mycobacterial cells, we used the method of fractionation of mycobacterial cultures into large and small aggregates, and single cells (Cougoule et al., 2002) and determined the percentages of cells (wet weight) that pelleted at $100 \mathrm{~g}$, i.e. larger clumps. Cells from strain TM99 were found to aggregate fivefold more than did the parent strain (Table 1). This phenomenon could be due to the change of the cell surface hydrophobicity, which is known to play a major role in the cellular adhesion of Corynebacterineae (Bendinger et al., 1993; Borrego et al., 2000; see also Goren \& Brennan, 1979), and useful information may be obtained with use of Congo red, a planar and hydrophobic diazo-dye that binds lipids, lipoproteins and a broad range of other macromolecules (Cangelosi et al., 1999). Consistent with an increase of the cell surface hydrophobicity, cells from strain TM99 bound twice as much dye as the $\mathrm{mc}^{2} 155$ strain (Table 1). As the Congo red binding assay was performed on non-fractionated cultures, the possibility existed that the observed difference above was, at least in part, due to a trapping of the dye in large cellular clumps of TM99 rather than a real cellular accumulation per se. To address this question, mycobacterial cultures grown for 3 days in the presence of Congo red were washed, and aliquots were sonicated for various time periods, to disrupt aggregates of variable sizes into single cells. The resulting cells were washed again until colourless supernatants were obtained; the dye was then extracted from single cells and the binding determined. Whatever the sonication times used (data not shown) similar amounts of dye per $\mathrm{mg}$ wet weight were consistently associated with cells; in addition, this phenomenon was independent of the proportion of aggregates in the suspensions. It was thus concluded that the observed Congo red accumulation likely reflected the surface properties of individual cells. To further ascertain the difference in hydrophobicity between the parent and mutant strains, the hexadecane partition procedure was used (Rosenberg et al., 1980). This rapid quantitative assay is based on the degree of interaction of cells with liquid hydrocarbon following a brief period of mixing. Again, cells from strain TM99 exhibited a hydrophobicity index significantly greater than that of the parent strain (Table 1); in a typical experiment, 


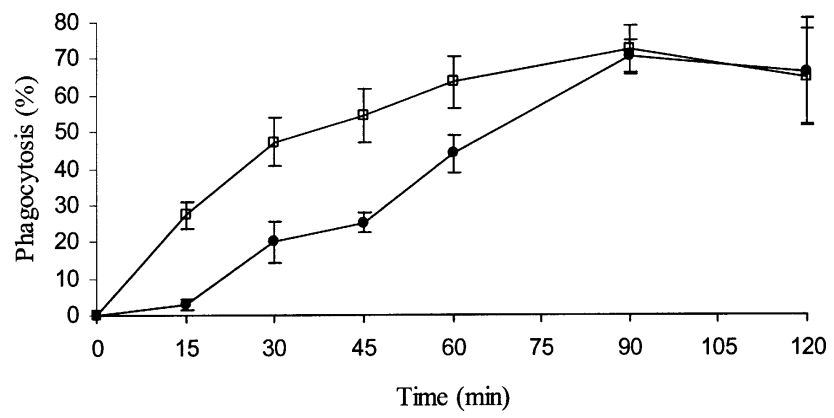

Fig. 3. Phagocytosis of the $m c^{2} 155(0)$ and the $m p s$-inactivated TM99 ( $\square$ ) strains of M. smegmatis by human MDMs. MDMs were exposed for various times to FITC-labelled mycobacteria (cell: bacteria ratio, 1:50), then washed and fixed. At least 100 cells per slide were counted under fluorescence microscopy. The percentage of phagocytic cells having ingested at least one bacterium is expressed as the mean $\pm S D$ of at least three independent experiments performed in duplicate.

$52.5 \%$ of cells from strain TM99 cells were extracted with hexadecane whereas only $27.9 \%$ of those from strain $\mathrm{mc}^{2} 155$ were recovered in the organic phase.

Most prokaryotic cells present a negative surface charge, a parameter that may contribute to the adhesive properties of cells (Van Loosdrecht et al., 1987; Bendinger et al., 1993). Although C-type GPLs of $M$. smegmatis are neutral lipids (Daffé et al., 1983), the absence of these molecules may induce a change in the distribution of surface-exposed lipids of the mutant, resulting in a relative abundance of phospholipids already present in the outermost compartment of the envelope (Ortalo-Magné et al., 1996) whose phosphate groups may influence the surface net charge of cells. Because electrophoresis allows the examination of large populations of microorganisms and has been demonstrated to be the most appropriate method among those experimentally examined for measuring the net charge of bacteria cells (Bayer \& Sloyer, 1990), strain TM99 and its parent strain were compared by this technique. No significant difference was found between the negative zeta-potential displayed by the two strains (Table 1).

\section{Internalization of the mps-disrupted mutant}

M. smegmatis is internalized by MDMs through receptors that include the mannose receptor (AstarieDequeker et al., 1999). To investigate the consequence of the phenotypic changes induced by the absence of Ctype GPLs from the surface of strain TM99 on the nonopsonic phagocytosis of the bacterial cells, the kinetics of internalization of individualized cells from the wild-type and mutant strains were compared (Fig. $3)$. A high rate of phagocytosis of both strains of $M$. smegmatis $(70 \%)$ was observed when bacterial cells were incubated with MDMs for 90 min. Interestingly, while only $5 \%$ of MDMs internalized the wild-type strain in the early $15 \mathrm{~min}$, mutant cells were phagocytosed by $40 \%$ of the MDMs during the same period; a significant difference in the uptake rate of individual bacterial cells from the two strains was still observed up to $1 \mathrm{~h}$ after the addition of bacteria. After this time period, however, the percentages of MDMs having internalized both types of mycobacterial cells were very similar. This observation indicated that the absence of C-type GPLs from the bacterial cell surface induced a very rapid internalization of $M$. smegmatis cells by MDMs.

\section{Composition of the extracellular material of the mps- inactivated mutant}

In most published chemical models of the mycobacterial cell envelope, type- and species-specific lipids such as GPLs are proposed to form a monolayer of lipid that covers the entire mycobacterial surface (Brennan \& Nikaido, 1995; Liu et al., 1995; Minnikin, 1982). One possible consequence of the absence of GPLs from the mutant cells may be the release of surface-exposed components of the mutant into the culture medium, assuming that GPLs play a crucial structural role in the architecture of the outermost layer of the parent strain; accordingly, the absence of C-type GPLs would cause a disorganization of the layer and the release of some of its components. To test this hypothesis, the composition of the extracellular material of the mutant was compared to that of the parent strain. The amounts of extracellular compounds in LB culture broth could not be determined, due to the high content of peptones and carbohydrate in this medium. Nevertheless, reasoning that the LB medium of strain TM99 should contain some lipids if the bacterial outermost constituents were released from the bacterial surface, the LB culture fluids from the mutant and parent strains were extracted with organic solvents and the organic phases were analysed. No significant amount of lipids was detected in these extracts. To compare the amounts of materials released into the culture fluids, the two strains were grown in synthetic Sauton's medium. Similar amounts of carbohydrate and protein were found in the culture fluids of the two strains which exhibited the same sugar composition (data not shown) that was similar to that previously found for M. smegmatis (Lemassu et al., 1996). Thus, the absence of GPLs from the mutant strain does not induce the release of surface components into the culture fluid.

\section{Composition of the SEM of the mps-disrupted mutant}

Even if GPLs are not important for the architecture of the outermost layer, they are assumed to be abundant and known to be surface-located. If so, the absence of GPLs from the surface of mutant cells should induce the exposure of the substances (such as trehalose dimycolates) that are known to be located in deeper compartments of the cell envelope of the parent strain (OrtaloMagné et al., 1996). To address this question, the nature and relative amounts of the SEM of the mutant were determined and compared to those of the parent strain (Table 2). Gentle shaking of the bacterial cells with glass beads, a technique that declumps aggregates by extract- 
Glycopeptidolipids and cell envelope of mycobacteria

Table 2. Lipid composition of the wild-type ( $\left.\mathrm{mc}^{2} 155\right)$ and mps-inactivated (TM99) strains of M. smegmatis

The lipid composition is shown as relative percentages of radioactivity incorporated into lipids.

\begin{tabular}{|lccccc|}
\hline \multirow{2}{*}{ Lipid } & \multicolumn{2}{c|}{ mc $^{2} \mathbf{1 5 5}$} & & \multicolumn{2}{c|}{ TM99 } \\
\cline { 2 - 5 } & $\begin{array}{c}\text { Bead-treated } \\
\text { bacterial lipids }\end{array}$ & $\begin{array}{c}\text { Surface-exposed } \\
\text { lipids }\end{array}$ & & $\begin{array}{c}\text { Bead-treated } \\
\text { bacterial lipids }\end{array}$ & $\begin{array}{c}\text { Surface-exposed } \\
\text { lipids }\end{array}$ \\
\hline Triacylglycerols & $13 \cdot 9$ & Trace & $84 \cdot 6$ & $16 \cdot 1$ & Trace \\
Glycopeptidolipids & $18 \cdot 3$ & $0 \cdot 0$ & $0 \cdot 0$ & $0 \cdot 0$ \\
Trehalose dimycolates & $8 \cdot 8$ & Trace & $10 \cdot 4$ & $0 \cdot 0$ \\
Trehalose monomycolates & $11 \cdot 2$ & $13 \cdot 3$ & $13 \cdot 1$ & Trace \\
Phospholipids & $47 \cdot 8$ & & $60 \cdot 8$ & $95 \cdot 0$ \\
\hline
\end{tabular}

ing the amorphous material covering cells as observed by scanning electron microscopy (Ortalo-Magné et al., 1995, 1996), was used. The amount of SEM extracted with glass beads from the surface of the mutant was similar to that obtained from the parent strain (15 to $16 \%$ of the cell dry weight). The carbohydrate and protein contents of the SEMs from the two strains were comparable. The percentages of carbohydrate in the SEMs of the parent and the mutant strains were estimated to $13.2 \%$ and $13.8 \%$ of the dry weight, respectively; the sugar compositions of the SEMs from both origins were similar and consisted of arabinose, mannose, xylose and glucose (Lemassu et al., 1996). Protein represented $59.3 \%$ and $75.4 \%$ of the dry weight of the SEMs of the parent and mutant strains, respectively. Surface lipids represent a very minor fraction $(0 \cdot 02-0 \cdot 1 \%$ bacterial dry weight) of mycobacteria grown on synthetic Sauton's medium (Lemassu et al., 1996; Ortalo-Magné et al., 1996). Consequently, bacterial cells of the two strains grown to exponential phase cells were labelled with sodium $\left[{ }^{14} \mathrm{C}\right]$ acetate before extracting SEMs to easily quantify surface-exposed lipids. In contrast, larger amounts of lipids were extracted from the SEMs of both strains grown on LB medium. They represented $3.4 \%$ and $2.3 \%$ bacterial dry weight of the parent and mutant strains, respectively. C-type GPLs represented $85 \%$ of the radioactivity found in the surface-exposed lipids from the wild-type strain grown on both media and were not detected in lipids from strain TM99 (Table 2), as expected. Lipids extracted from the parent and mutant strains after bead treatment represented 10.5 and $11.2 \%$ of the cell dry weight, respectively. The lipid composition of the materials originated from both strains was similar, except for the absence of C-type GPLs, and consisted of triacyl glycerols, trehalose monomycolates, trehalose dimycolates and phospholipids. Importantly, it has to be noted that trehalose dimycolates, which were not detected in the surface-exposed lipids from the parent strain, were also absent from the SEM of strain TM99. This data indicated that the absence of GPLs from the mutant strain does not induce the exposure of lipids that are buried in deeper compartments of the cell envelope.

GPLs represented the major lipid constituents of the parent strain of M. smegmatis grown on LB both (Table 2); they remained a prominent class of lipids of bacterial cells grown in the Sauton's medium, although more triacyl glycerols were produced by bacteria grown on this glycerol-rich medium (data not shown). Based on the relative percentages of labelled lipids and the amounts of lipids extracted from SEMs it was estimated that $50 \%$ of GPLs were localized in the outermost layer of the parent strain of M. smegmatis grown in LB medium. The remaining GPLs were present in deeper compartments of the cell envelope of the parent strain and extracted after bead treatment of the cells with organic solvents.

\section{Ultrastructural features of the mps-inactivated strain TM99}

Cells from M. smegmatis strain $\mathrm{mc}^{2} 155$ and its isogenic TM99 mutant were grown in both LB and Sauton's media and examined by electron microscopy (Fig. 4). Ultrathin sections of cells were stained with ruthenium red, a stain that strongly reacts with the surface of mycobacteria (Rastogi et al., 1984). Examination of thin sections revealed a cell envelope structure of $M$. smegmatis strain $\mathrm{mc}^{2} 155$ (Fig. 4A) composed of (i) a plasma membrane, (ii) a thick internal electron-dense layer, (iii) an electron-transparent layer and (iv) a thick electrondense outer layer $(\mathrm{OL})$; the space observed between the plasma membrane and the thick electron-dense layer corresponds to the hypothetical periplasmic space (Daffé \& Draper, 1998). This ultrastructural appearance is similar to that previously found in other mycobacterial species (Daffé \& Draper, 1998; Draper, 1982; Paul \& Beveridge, 1992; Rastogi et al., 1986). In sharp contrast, the thick electron-dense OL stained with ruthenium red and observed on the thin sections of the parent $M$. smegmatis strain $\mathrm{mc}^{2} 155$ (Fig. 4A) was unlabelled in those of the cells from the isogenic mps-inactivated strain TM99 (Fig. 4B). However, the thickness of the electron-transparent layer of the mutant roughly corresponded to the sum of the thickness of the electrontransparent layer and that of the OL of the parent strain. These data suggested that the absence of staining of the OL of the mutant strain was not due to the loss of this layer but, rather, may be attributed to absence of GPLs 

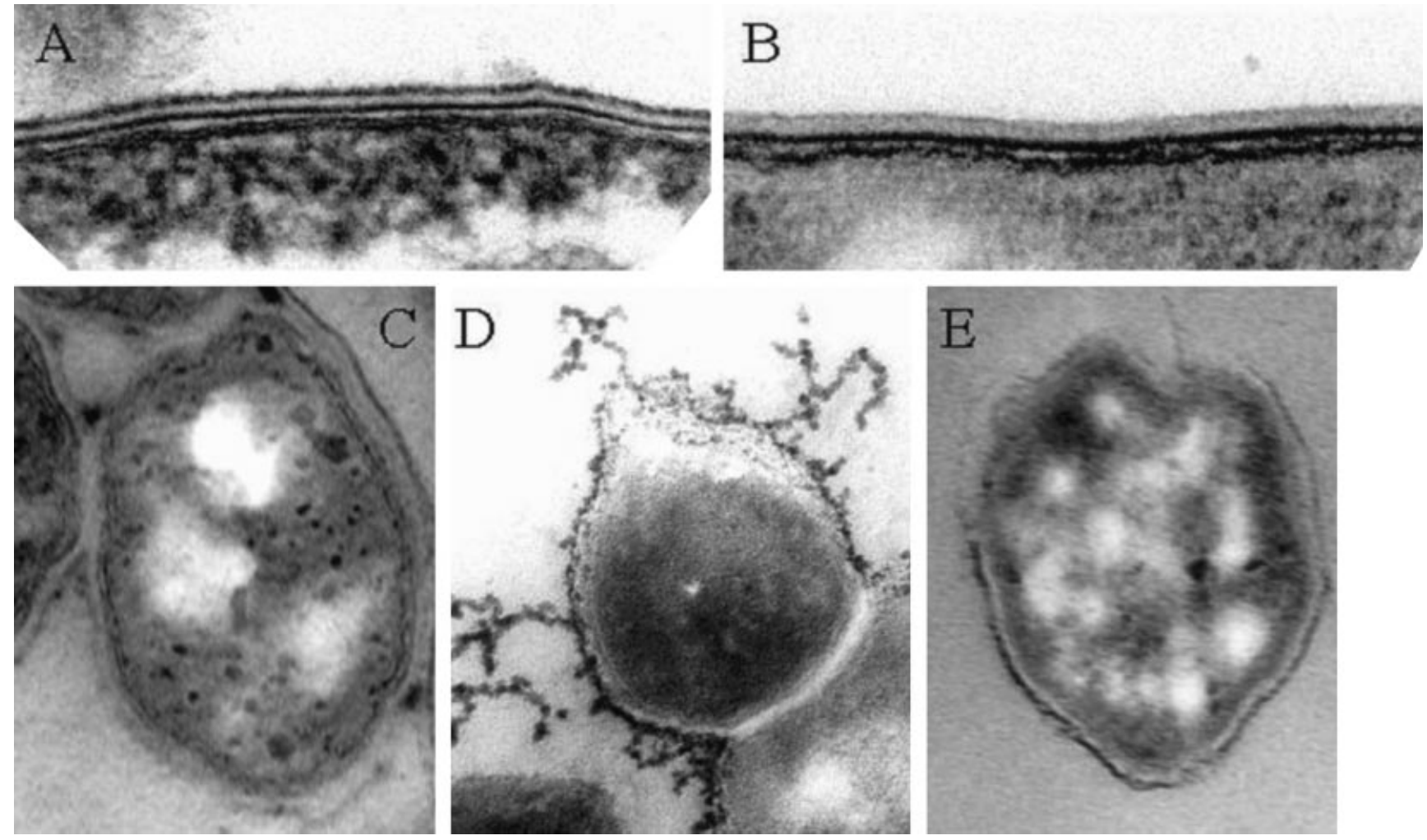

Fig. 4. Transmission electron micrographs of $M$. smegmatis strain $m c^{2} 155(A, D$ and $E$ ) and its isogenic mps-inactivated strain TM99 (B and C). Strains were grown either in LB ( $A, B$ and $D)$ or Sauton's medium ( $C$ and $E)$.

from the surface of the mutant cells which would bind ruthenium red. This conclusion was further supported by the observation of the thickness of this layer in adjacent cells (Fig. 4C). That the staining of OL in the parent strain by ruthenium red is attributable to GPLs is supported by the correlation between the ultrastructural appearance of stained cells and the amounts of surfaceexposed GPLs. When large amounts of GPLs are present on the cell surface of the parent strain (growth in the LB medium, see above), the whole surface of the bacilli is stained by the dye that also interacts with GPLs budding from the cell surface and with an appearance of filamentous and rope-like structures (Fig. 4D). When cells exposed less amounts of GPLs on their surface (growth in Sauton's medium, see above) they are stained less by ruthenium red (Fig. 4E). These data establish that the dye binds to the OL through interactions with surface-exposed GPLs.

\section{Cell wall permeability of the mutant}

Because GPLs are major lipids of M. smegmatis (Table 2) and at least $50 \%$ of GLPs are not surface-exposed but located in deeper compartments of the cell envelope, it was interesting to address the question of the possible implication of C-type GPLs in the cell wall permeability barrier. To determine whether this barrier was altered in the mps-inactivated mutant of M. smegmatis, the uptake of the hydrophobic chenodeoxycholate by cells of the

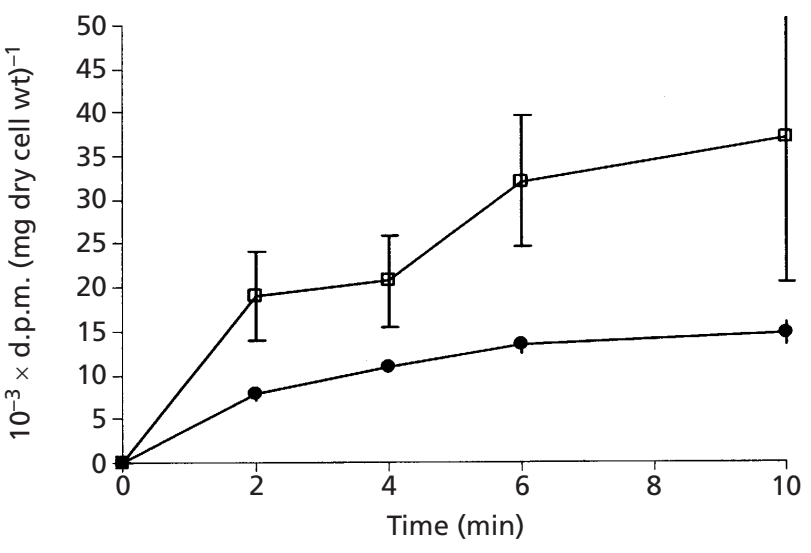

Fig. 5. $\left[{ }^{14} \mathrm{C}\right]$-Chenodeoxycholate uptake by the $\mathrm{mc}^{2} 155(0)$ and the mps-inactivated TM99 ( $\square)$ strains of $M$. smegmatis. Exponential-phase cells were first incubated overnight with $\left[5,6-{ }^{3} \mathrm{H}\right]$ uracil to quantify the biomass present in aliquots used in the accumulation assays. Accumulation assays of $\left[{ }^{14} \mathrm{C}\right]$ chenodeoxycholate were performed in $1 \mathrm{ml}$ HEPES buffer under continuous agitation; aliquots were taken at different time intervals and counting (in d.p.m.) was performed. The values are the mean $\pm S D$ of at least three independent experiments.

wild-type strain $\mathrm{mc}^{2} 155$ and the TM99 mutant were compared (Fig. 5). This probe is a negatively charged hydrophobic molecule that has been previously used to 
evaluate the fluidity of the mycobacterial cell wall lipids (Liu et al., 1996; Yuan et al., 1997). The mutant showed a significantly higher initial rate of uptake and accumulation of chenodeoxycholate than did the parent strain. This result indicated that C-type GPLs contribute to the permeability barrier of the cell wall of $M$. smegmatis.

The permeability of the cell wall will determine whether certain antibiotics can enter bacteria and achieve their inhibitory action; we therefore investigated the susceptibility of strain TM99 and its parent to various molecules. The MICs of the molecules examined were unaffected by the mutation; these included the relatively hydrophobic rifampicin $\left(256 \mu \mathrm{g} \mathrm{ml}^{-1}\right)$, chloramphenicol $\left(32 \mu \mathrm{g} \mathrm{ml}^{-1}\right)$ and novobiocin $\left(32 \mu \mathrm{g} \mathrm{ml}^{-1}\right)$, and the hydrophilic hygromycin B $\left(64 \mu \mathrm{g} \mathrm{ml}^{-1}\right)$, isoniazid $\left(4 \mu \mathrm{g} \mathrm{ml}^{-1}\right)$ and streptomycin $\left(2 \mu \mathrm{g} \mathrm{ml}^{-1}\right)$. These data indicated that the diffusion of these antibiotics through the cell wall barrier was not the limiting step for their activity. A similar observation has been made for the fbpC-inactivated mutant of Mycobacterium tuberculosis, which exhibited a change in its cell wall permeability to chenodeoxycholate due to a $40 \%$ reduction of covalently linked mycolates (Jackson et al., 1999).

\section{DISCUSSION}

The present study addressed the question of the function of C-type GPLs, one of the major species- or typespecific lipid constituents of the cell envelopes of many non-tuberculous mycobacteria (Brennan, 1988; Daffé \& Lemassu, 2000), in the physiology of these bacteria. For this purpose, a mutant derived from M. smegmatis $\mathrm{mc}^{2} 155$ by transposon insertion mutagenesis, strain TM99, and previously shown to be defective in the production of C-type GPLs (Billman-Jacobe et al., 1999) was analysed. Genetic analysis has revealed that the mutation in TM99 was the result of the insertion of the transposon in the mps gene encoding a mycobacterial nonribosomal peptide synthase. The same phenotype has been found for two insertional mutants of $M$. smegmatis $\mathrm{mc}^{2} 155$, namely strains $9-1045$ and 8-245 (Billman-Jacobe et al., 1999; Recht et al., 2000). While the transposon was localized in the mps gene in strain 9-1045, in strain 8-245 the mutation occurred in the tmtpC ORF encoding a putative transport membrane protein (Recht et al., 2000). The tmtpC-disrupted mutant has been shown by labelling experiments to be unable to synthesize GPLs, suggesting that the encoded protein may be involved, not only in the transport of the glycolipids, but also in the production of GPLs (Recht et al., 2000). Compared to the wild-type smooth morphotype strain, the C-type-GPL-deficient mutant strains TM99, 9-1045 and 8-245 exhibited a rough colony morphology (this work, Recht et al., 2000). This observation was in agreement with previous studies on GPL-deficient $M$. avium-intracellulare (Barrow \& Brennan, 1982; Belisle et al., 1991). The absence of C-type GPLs also affects the surface properties of strain TM99. This mutant strain, as well as strains 9-1045 and 8-245 (this work, Recht et al., 2000), was found to display a spreading halo smaller than that of the parent $\mathrm{mc}^{2} 155$ strain, but similar to those of the other C-type GPL-negative mutants. In addition, the cell hydrophobicity of the mutants were significantly higher than that of their parent strain. As a consequence, the three mutant strains also exhibited identical macroscopic aspects, both in shaken and static cultures. A similar observation has been made for strains of Gordona devoid of a specific glycolipid; colonies of these latter strains exhibited a rough morphology and were composed of highly aggregated cells possessing a more hydrophobic cell surface than the smooth morphotypes (Moormann et al., 1997).

In M. smegmatis, the phenotypic changes were associated with the absence of binding of ruthenium red to the surface of the mutant cells, as observed by transmission electron microscopy. This data implies that the surfaceexposed C-type GPLs of M. smegmatis are the substances that react with ruthenium red to give the electron-dense appearance of the outermost cell envelope layer. The absence of these compounds, however, does not cause the disorganization of the outer layer since no specific release of surface constituents into the culture broth of the mutant was observed. Consistent with this observation, compounds such as trehalose dimycolates that are located in deeper compartments of the cell envelope of the parent strain (Ortalo-Magné et al., 1996) were not surface-exposed in the mutant. These data reinforce the credibility of the recent model (Daffé \& Draper, 1998) in which the surface of mycobacteria is composed of polysaccharides, proteins and lipids, as opposed to models in which the surface consists of a lipid layer (Brennan \& Nikaido, 1995; Liu et al., 1995; Minnikin, 1982). Nevertheless, at the interface between the bacterial and host cells, surface-exposed GPLs may play a role in the internalization of C-type GPL-containing non-tuberculous mycobacteria by macrophages which are believed to use glycoconjugates as ligands of many of their receptors (Ehlers \& Daffé, 1998). Accordingly, phagocytosis of the wild-type strain and its isogenic mps-inactivated strain of M. smegmatis by human MDMs was investigated. As expected from the existence of numerous receptors capable of internalizing mycobacteria, both strains were found in phagosomes but the kinetics of phagocytosis of the mutant was much more rapid than that of the parent strain. This result suggests that either GPLs may mask some as yet undefined ligands that interact with highly efficient macrophage receptor(s) or, alternatively, the bacterial cell surface hydrophobicity may play an important role in the rate of internalization of bacteria. Further studies are warranted to determine the precise role of GPLs in the internalization of mycobacteria.

GPLs are the major extractable lipids of M. smegmatis and other non-tuberculous mycobacterial species containing these molecules and a large portion of these substances are localized in the deeper compartments of the wild-type M. smegmatis envelope, presumably in the mycolic acid-containing asymmetric bilayer. It was therefore important to investigate the consequence of the absence of GPLs on cell wall architecture, especially 
its implication in the outer permeability barrier. In all currently proposed models (Brennan \& Nikaido, 1995; Daffé \& Draper, 1998; Liu et al., 1995; Minnikin, 1982; Rastogi, 1991) the outer permeability barrier of mycobacteria consists of a monolayer of mycoloyl residues covalently linked to the cell wall arabinogalactan and includes other lipids which are probably arranged to form a bilayer with the mycoloyl residues. Although no sign of a second lipid bilayer has ever been reported in thin sections of mycobacterial cells (Draper, 1998), freeze-fractured samples of mycobacteria (Barksdale \& Kim, 1977; Benedetti et al., 1984; Takeo et al., 1984) showed that these organisms had two such planes of weakness in their envelopes; in addition to the expected plasma membrane fracture, a second fracture plane, close to the cell surface of mycobacteria, was observed. The cell wall-linked mycolates certainly participate in this barrier since the disruption of a gene that encodes a mycoloyltransferase, namely antigen-85C, causes a decrease in the amount of cell wall-bound mycolates and affects the permeability of the envelope of the mutant (Jackson et al., 1999). Evidence has also been presented that the chemical structure of mycolic acids plays a role in determining the fluidity and permeability of the mycobacterial cell wall (George et al., 1995; Liu et al., 1996; Dubnau et al., 2000). To date, however, the implication of non-covalently bound lipids in the wall bilayer has been demonstrated for only phthiocerol dimycocerosates of M. tuberculosis (Camacho et al., 2001). The present work demonstrated that the absence of C-type GPLs from the cell envelope of M. smegmatis has a profound effect on the uptake of chenodeoxycholate, a hydrophobic molecule that diffuses through lipid domains of the mycobacterial cell wall (Liu et al., 1996; Yuan et al., 1997). Based on the localization of cell wall fracture plane in mycobacteria (Barksdale \& Kim, 1977; Benedetti et al., 1984; Takeo et al., 1984), far from the cell surface, it is likely that the GPLs located in inner compartments of the cell envelope, but not surfaceexposed GPLs, contribute to the permeability barrier.

In conclusion, in addition to be distinctive markers of numerous mycobacterial species that may help in the diagnosis of non-tuberculous diseases and for taxonomic purposes, GPLs are also involved in the wall permeability barrier that certainly plays a role in bacterial physiology.

\section{ACKNOWLEDGEMENTS}

We thank Dr J. Recht for providing mutant strains 8-245 and 9-1045 of M. smegmatis for comparative phenotypic studies and Mrs Khim Hoe for expert assistance in electron microscopy.

\section{REFERENCES}

Astarie-Dequeker, C., N'Diaye, E. N., Le Cabec, V., Rittig, M. G., Prandi, J. \& Maridonneau-Parini, I. (1999). The mannose receptor mediates uptake of pathogenic and nonpathogenic mycobacteria and bypasses bactericidal responses in human macrophages. Infect Immun 67, 469-477.

Bardou, F., Raynaud, C., Ramos, C., Lanéelle, M.-A. \& Lanéelle, G.
(1998). Isoniazid uptake mechanism in Mycobacterium tuberculosis. Microbiology 144, 2539-2544.

Barksdale, L. \& Kim, K. S. (1977). Mycobacterium. Bacteriol Rev 41, 217-372.

Barrow, W. W. \& Brennan, P. J. (1982). Isolation in high frequency of rough variants of Mycobacterium intracellulare lacking Cmycoside glycopeptidolipid antigens. J Bacteriol 150, 381-384.

Bayer, M. E. \& Sloyer, J. L. (1990). The electrophoretic mobility of Gram-negative and Gram-positive bacteria: an electrokinetic analysis. J Gen Microbiol 136, 867-874.

Belisle, J. T., Pascopella, L., Inamine, J. M., Brennan, P. J. \& Jacobs, W. R. (1991). Isolation and expression of a gene cluster responsible for biosynthesis of the glycopeptidolipid antigens of Mycobacterium avium. J Bacteriol 173, 6991-6997.

Bendinger, B., Rijnaarts, H. H. M., Altendorf, K. \& Zehnder, A. J. B. (1993). Physicochemical cell surface and adhesive properties of coryneform bacteria related to the presence and chain length of mycolic acids. Appl Environ Microbiol 59, 3973-3977.

Benedetti, E. L., Dunia, I., Ludosky, M. A., Nguyen, V. M., Dang, D. T., Rastogi, N. \& David, H. L. (1984). Freeze-etching and freezefracture structural features of cell envelopes in mycobacteria and leprosy derived corynebacteria. Acta Leprol 2, 237-248.

Besra, G. S., McNeil, M. R., Rivoire, B., Khoo, K. H., Morris, H. R., Dell, A. \& Brennan, P. J. (1993). Further structural definition of a new family of glycopeptidolipids from Mycobacterium xenopi. Biochemistry 32, 347-355.

Billman-Jacobe, H., McConville, M. J., Haites, R. E., Kovacevic, S. \& Coppel, R. L. (1999). Identification of a peptide synthetase involved in the biosynthesis of glycopeptidolipids of Mycobacterium smegmatis. Mol Microbiol 33, 1244-1253.

Borrego, S., Niubó, E., Ancheta, O. \& Espinosa, M. E. (2000). Study of the microbial aggregation in Mycobacterium using image analysis and electron microscopy. Tissue Cell 32, 494-500.

Brennan, P. J. (1988). Mycobacterium and other actinomycetes. In Microbial Lipids, vol. 1, pp. 203-298. Edited by C. Ratledge \& S. G. Wilkinson. London: Academic Press.

Brennan, P. J. \& Nikaido, H. (1995). The envelope of mycobacteria. Annu Rev Biochem 64, 29-63.

Camacho, L. R., Constant, P., Raynaud, C., Lanéelle, M.-A., Triccas, J. A., Gicquel, B., Daffé, M. \& Guilhot, C. (2001). Analysis of the phthiocerol dimycocerosate locus of Mycobacterium tuberculosis: evidence that this lipid is involved in the cell wall permeability barrier. J Biol Chem 276, 19845-19854.

Cangelosi, G. A., Palermo, C. O., Laurent, J.-P., Hamlin, A. M. \& Brabant, W. H. (1999). Colony morphotypes on Congo red agar segregate along species and drug susceptibility lines in the Mycobacterium avium-intracellulare complex. Microbiology 145, 1317-1324.

Cougoule, C., Constant, P., Etienne, G., Daffé, M. \& MaridonneauParini, I. (2002). Lack of fusion of azurophil granules with phagosomes during phagocytosis of Mycobacterium smegmatis by human neutrophils is not actively controlled by the bacteria. Infect Immun 70, 1591-1598.

Daffé, M. \& Draper, P. (1998). The envelope layers of mycobacteria with reference to their pathogenicity. Adv Microbiol Physiol 39, 131-201.

Daffé, M. \& Lemassu, A. (2000). Glycobiology of the mycobacterial surface: structure and biological activities of the cell envelope glycoconjugates. In Glycomicrobiology, pp. 225-273. Edited by R. J. Doyle. New York: Kluwer/Plenum.

Daffé, M., Lanéelle, M.-A. \& Puzo, G. (1983). Structural elucidation by field desorption and electron-impact mass spec- 
trometry of the C-mycosides isolated from Mycobacterium smegmatis. Biochim Biophys Acta 751, 439-443.

Dische, Z. (1962). Color reaction of hexoses. Methods Carbohydr Chem 1, 488-494.

Dittmer, J. C. F. \& Lester, R. L. (1964). A simple specific spray for the detection of phospholipids on thin layer chromatograms. J Lipid Res 5, 126-127.

Draper, P. (1974). The mycoside capsule of Mycobacterium avium 357. J Gen Microbiol 83, 431-433.

Draper, P. (1982). The anatomy of mycobacteria. In The Biology of The Mycobacteria, vol. 1, Physiology, Identification and Classification, pp. 9-49. Edited by C. Ratledge \& J. L. Stanford. London: Academic Press.

Draper, P. (1998). The outer parts of the mycobacterial envelope as permeability barrier. Frontiers Biosci 3, 1253-1261.

Dubnau, E., Chan, J., Raynaud, C., Mohan, V. P., Lanéelle, M.-A., Yu, K., Quemard, A., Smith, I. \& Daffé, M. (2000). Oxygenated mycolic acids are necessary for virulence of Mycobacterium tuberculosis in mice. Mol Microbiol 36, 630-637.

Ehlers, M. R. \& Daffé, M. (1998). Interactions between Mycobacterium tuberculosis and host cells: are mycobacterial sugars the key? Trends Microbiol 6, 328-335.

Furuchi, A. \& Tokunaga, T. (1972). Nature of the receptor substance of Mycobacterium smegmatis for D4 bacteriophage adsorption. J Bacteriol 111, 404-411.

George, K. M., Yuan, Y., Sherman, D. R. \& Barry, C. E. (1995). The biosynthesis of cyclopropanated mycolic acids in Mycobacterium tuberculosis: identification and functional analysis of cma-2. J Biol Chem 270, 27292-27298.

Goren, M. B. \& Brennan, P. J. (1979). Mycobacterial lipids: chemistry and biological activities. In Tuberculosis, pp. 63-193. Edited by G. P. Youmans. Philadelphia, PA: WB Saunders.

Goren, M. B., McClatchy, J. K., Martens, B. \& Brokl, O. (1972). Mycosides C: behavior as receptor site substance for mycobacteriophage D4. J Virol 9, 999-1003.

Jackson, M., Raynaud, C., Lanéelle, M.-A., Guilhot, C., LaurentWinter, C., Ensergueix, D., Gicquel, B. \& Daffé, M. (1999). Inactivation of the antigen $85 \mathrm{C}$ gene profoundly affects the mycolate content and alters the permeability of the Mycobacterium tuberculosis cell envelope. Mol Microbiol 31, 1573-1587.

Lemassu, A., Ortalo-Magné, A., Bardou, F., Silve, G., Lanéelle, M.-A. \& Daffé, M. (1996). Extracellular and surface-exposed polysaccharides of non-tuberculous mycobacteria. Microbiology 142, 1513-1520.

Liu, J., Rosenberg, E. Y. \& Nikaido, H. (1995). Fluidity of the lipid domain of cell wall from Mycobacterium chelonae. Proc Natl Acad Sci U S A 92, 11254-11258.

Liu, J., Barry, C. E., Besra, G. S. \& Nikaido, H. (1996). Mycolic acid structure determines the fluidity of the mycobacterial cell wall. J Biol Chem 271, 29545-29551.

Martinez, A., Torello, S. \& Kolter, R. (1999). Sliding motility in mycobacteria. J Bacteriol 181, 7331-7338.

Minnikin, D. E. (1982). Lipids: complex lipids, their chemistry, biosynthesis and roles. In The Biology of the Mycobacteria, vol 1, Physiology, Identification and Classification, pp. 95-184. Edited by C. Ratledge \& J. L. Stanford. London: Academic Press.

Moormann, M., Zähringer, U., Moll, H., Kaufmann, R., Schmid, R. \& Altendorf, K. (1997). A new glycosylated lipopeptide incorporated into the cell wall of a smooth variant of Gordona bydrophobica. J Biol Chem 272, 10729-10738.

Mukhopadhyay, S., Basu, D. \& Chakrabarti, P. (1997). Characteri- zation of a porin from Mycobacterium smegmatis. J Bacteriol 179, 6205-6207.

N’Diaye, E. N., Darzacq, X., Astarie-Dequeker, C., Daffé, M., Calafat, J. \& Maridonneau-Parini, I. (1998). Fusion of azurophil granules with phagosomes and activation of the tyrosine kinase Hck are specifically inhibited during phagocytosis of mycobacteria by human neutrophils. J Immunol 161, 4983-4991.

Ortalo-Magné, A., Dupont, M.-A., Lemassu, A., Andersen, A. B., Gounon, P. \& Daffé, M. (1995). Molecular composition of the outermost capsular material of the tubercle bacillus. Microbiology 141, 1609-1620.

Ortalo-Magné, A., Lemassu, A., Lanéelle, M.-A., Bardou, F., Silve, G., Gounon, P., Marchal, G. \& Daffé, M. (1996). Identification of the surface-exposed lipids on the cell envelope of Mycobacterium tuberculosis and other mycobacterial species. J Bacteriol 178, 456-461.

Paul, T. R. \& Beveridge, T. J. (1992). Reevaluation of envelope profiles and cytoplasmic ultrastructure of mycobacteria processed by conventional embedding and freeze-substitution protocols. J Bacteriol 174, 6508-6517.

Peyron, P., Bordier, C., N'Diaye, E. N. \& Maridonneau-Parini, I. (2000). Nonopsonic phagocytosis of Mycobacterium kansasii by human neutrophils depends on cholesterol and is mediated by CR3 associated with glycosylphosphatidylinositol-anchored proteins. J Immunol 165, 5186-5191.

Rastogi, N. (1991). Recent observations concerning structure and function relationships in the mycobacterial cell envelope: elaboration of a model in terms of mycobacterial pathogenicity, virulence and drug-resistance. Res Microbiol 142, 464-476.

Rastogi, N., Fréhel, C. \& David, H. L. (1984). Cell envelope architectures of leprosy-derived corynebacteria, Mycobacterium leprae, and related organisms: a comparative study. Curr Microbiol 11, 23-30.

Rastogi, N., Fréhel, C. \& David, H. L. (1986). Triple-layered structure of mycobacterial cell wall: evidence for the existence of a polysaccharide-rich outer layer in 18 mycobacterial species. Curr Microbiol 13, 237-242.

Raynaud, C., Etienne, G., Peyron, P., Lanéelle, M.-A. \& Daffé, M. (1998). Extracellular enzyme activities potentially involved in the pathogenicity of Mycobacterium tuberculosis. Microbiology 144 , 577-587.

Recht, J., Martinez, A., Torello, S. \& Kolter, R. (2000). Genetic analysis of sliding motility in Mycobacterium smegmatis. J Bacteriol 182, 4348-4351.

Rivière, M. \& Puzo, G. (1991). A new type of serine-containing glycopeptidolipid from Mycobacterium xenopi. J Biol Chem 266, 9057-9063.

Rosenberg, M., Gutnick, D. \& Rosenberg, E. (1980). Adherence of bacteria to hydrocarbons: a simple method for measuring cellsurface hydrophobicity. FEMS Microbiol Lett 9, 29-33.

Rulong, S., Aguas, A. P., da Silva, P. P. \& Silva, M. T. (1991). Intramacrophagic Mycobacterium avium bacilli are coated by a multiple lamellar structure: freeze fracture analysis of infected mouse liver. Infect Immun 59, 3895-3902.

Senaratne, R. H., Mobasheri, H., Papavinasasundaram, K. G., Jenner, P., Lea, E. J. A. \& Draper, P. (1998). Expression of gene for a porin-like protein of the OmpA family from Mycobacterium tuberculosis H37Rv. J Bacteriol 180, 3541-3547.

Takeo, K., Kimura, K., Kuze, F., Nakai, E., Nonaka, T. \& Nishiura, M. (1984). Freeze-fracture observations on the cell walls and peribacillary substances of various mycobacteria. J Gen Microbiol 130, 1151-1159. 
Tereletsky, M. J. \& Barrow, W. W. (1983). Postphagocytic detection of glycopeptidolipids associated with the superficial L1 layer of Mycobacterium intracellulare. Infect Immun 41, 13121321.

Trias, J. \& Benz, R. (1994). Permeability of the cell wall of Mycobacterium smegmatis. Mol Microbiol 14, 283-290.

Trias, J., Jarlier, V. \& Benz, R. (1992). Porins in the cell wall of mycobacteria. Science 258, 1479-1481.

Van Loosdrecht, M. C. M., Lyklema, J., Norde, W., Schraa, G. \&
Zehnder, A. J. B. (1987). Electrophoretic mobility and hydrophobicity as a measure to predict the initial steps of bacterial adhesion. Appl Environ Microbiol 53, 1898-1901.

Yuan, Y., Crane, D. C., Musser, J. M., Sreevatsan, S. \& Barry, C. E., III (1997). MMAS-1, the branch point between cis- and transcyclopropane-containing oxygenated mycolates in Mycobacterium tuberculosis. J Biol Chem 272, 10041-10049.

Received 19 March 2002; revised 3 June 2002; accepted 6 June 2002 